\title{
Nonlinear entanglement growth in inhomogeneous space-times
}

\author{
Arkadiusz Kosior and Markus Heyl \\ Max-Planck-Institut für Physik Komplexer Systeme, Nöthnitzer Strasse 38, D-01187, Dresden, Germany
}

(Received 1 June 2020; accepted 17 September 2020; published 7 October 2020)

\begin{abstract}
Entanglement has become central for the characterization of quantum matter both in and out of equilibrium. In a dynamic context, entanglement exhibits universal linear temporal growth in generic systems, which stems from the underlying linear light cones as they occur in planar geometries. Inhomogeneous space-times can lead, however, to strongly bent trajectories. While such bent trajectories crucially impact correlation spreading and therefore the light-cone structure, it has remained elusive how this influences the entanglement dynamics. In this work, we investigate the real-time evolution of the entanglement entropy in one-dimensional quantum systems after quenches that change the underlying space-time background of the Hamiltonian. Concretely, we focus on the Rindler space describing the space-time in close vicinity to a black hole. As a main result, we find that entanglement grows sublinearly in a generic fashion both for interacting and noninteracting quantum matter. We further observe that the asymptotic relaxation becomes exponential, as opposed to algebraic for planar Minkowski space-times, and in the vicinity of the black hole, the relaxation time for large subsystems becomes independent of the subsystem size. We study entanglement dynamics both for the case of noninteracting fermions, allowing for exact numerical solutions, and for random unitary circuits representing a paradigmatic class of ergodic systems.
\end{abstract}

DOI: 10.1103/PhysRevResearch.2.043036

\section{INTRODUCTION}

Quantum entanglement [1] is a key feature of quantum systems and has become a cornerstone of many modern branches of physics, such as quantum information [2] (in particular, quantum cryptography [3-5], quantum teleportation $[6,7]$, quantum computing [8-12]) and quantum many-body physics $[13,14]$. In the latter, the importance of entanglement is reflected by its universal properties, both under equilibrium and nonequilibrium conditions. Ground states of gapped one-dimensional quantum systems obey an area law [15-17]. In nonequilibrium, the entanglement entropy of generic systems shows generally a linear growth in time, which is today explained through a semiclassical picture of ballistic quasiparticle propagation [18-31]. Although the applicability of the quasiparticle picture has been confirmed for a range of integrable models, including inhomogeneous initial states [32-35], this picture cannot be directly applied when the post-quench Hamiltonian is not translationally invariant. A prominent example is the logarithmic spreading of entanglement in a many-body localized phase [36-40] in disordered models. These models are, however, constructed on flat homogeneous space-time backgrounds and therefore their local properties are the same everywhere. It has remained, however,

Published by the American Physical Society under the terms of the Creative Commons Attribution 4.0 International license. Further distribution of this work must maintain attribution to the author(s) and the published article's title, journal citation, and DOI. Open access publication funded by the Max Planck Society. an open question how entanglement grows in spatially inhomogeneous systems due to inhomogeneity of the space-time itself.

In this work, we investigate the entanglement entropy growth generated by a quench of the underlying space-time metric of the Hamiltonian. As a prototypical example of an inhomogeneous space-time, we choose a Rindler space, which can be viewed an asymptotic space-time near a black hole horizon [41-43]. We find that in the case of a global inhomogeneous quench, the initial entanglement entropy growth is sublinear and attains a constant size-independent value in a long-time limit. This is the most noticeable difference in comparison to the translationally invariant case where the entanglement entropy growth is linear. We contend that this behavior is universal applying for both integrable and ergodic systems. We find that some important aspects of the physical picture can be captured qualitatively via semiclassical arguments based on the maximal speed of correlation propagation. The correlations spread within bent light cones defined by null geodesic of the $(1+1)$ Rindler metric which suggests that our results capture the universal properties of the continuum theory.

This papers is organized as follows. In Sec. II, we discuss the basic concepts which are central to this study and introduce the model. In Sec. III, we calculate the dynamics of correlations after a Rindler quench and draw semiclassical arguments that are helpful to understand some physical aspects of the entanglement evolution. In Sec. IV, we present numerical results for finite subsystems and, by achieving a data collapse, we identify size-independent universal behavior of entanglement. While in Secs. II-IV, we focus on the case of free fermions on the $1 \mathrm{D}$ lattice, Sec. $\mathrm{V}$ is devoted to random 
unitary circuits [44-47] that represent minimally structured ergodic models. We estimate the coarse grained entanglement entropy, which is equivalent to the minimal membrane description [45], and show the remarkable qualitative agreement of results for integrable and ergodic systems. In Sec. VI, we conclude.

\section{THE MODEL AND SETUP}

\section{A. Entanglement entropy}

Quantum entanglement has developed into a central concept in quantum many-body physics. One of the key consequence for a state $|\Psi\rangle$ to be entangled is that it cannot be written as a simple product of states belonging to different subsystems. Quantification of entanglement is possible through various entanglement measures [48]. In this work, we want to study the entanglement entropy defined as

$$
S=-\operatorname{Tr}_{A}\left[\hat{\rho}_{A} \ln \hat{\rho}_{A}\right],
$$

where $\hat{\rho}_{A}=\operatorname{Tr}_{B}|\Psi\rangle\langle\Psi|$ is a reduced density matrix of a subsystem $A$ traced over the rest of the system, a subsystem $B$.

Entanglement between two spatial intervals can be generated dynamically through a quantum quench when a system is prepared in an initial state which is not an eigenstate of the post-quench Hamiltonian. Calabrese and Cardy [18] have shown by path integral methods of $(1+1)$ quantum field theory that, up to a time $t^{*} \propto L / 2$, the entanglement entropy growths linearly with a rate independent of the subsystem size $L$ and attains a constant value $\propto L$ afterwards, yielding a volume law, which is due to the finite subsystem size.

The linear spreading of entanglement entropy turns out to be very general and holds in various noninteracting [19-25] and short-range interacting [26-30] models, also for inhomogeneous initial states [32,33]. (However, deviations from the linear growth can be found in a presence of Markovian bath [49].) The linear growth of entanglement is due to the maximal speed of information. In the $(1+1)$ Minkowski space-time

$$
d s^{2}=-c^{2} d t^{2}+d x^{2},
$$

the rate of any information propagation is bounded by the speed of light $c$ and all particle trajectories lie within a light cone defined by null geodesic. In the lattice systems, the role of the speed of light $c$ plays the Lieb-Robinson velocity [50], being the emergent maximal velocity of correlation spreading.

\section{B. Rindler metric}

Although the entanglement dynamics in spatially invariant quantum systems seems to be completely understood, the entanglement dynamics in inhomogeneous space-times is a completely open question. (However, driven inhomogeneous systems have been already investigated $[51,52]$.) In this paper, we study the entanglement evolution in systems on a Rindler background described by a $(1+1)$ metric [41-43]

$$
d s^{2}=-x^{2} d t^{2}+d x^{2},
$$

which serves us as a nontrivial example of an inhomogeneous metric. In the literature, the Rindler metric appears primarily in two related contexts. First of all, the Rindler metric is an asymptotic Schwarzschild metric [53] in the vicinity of a black hole horizon at $x=0$. Secondly, the Rindler metric describes a flat Minkowski space-time in a hyperbolically accelerated reference frame, i.e., it characterizes the motion of uniformly accelerated observers [54].

Comparing Minkowski and Rindler metrics, one could interpret $c(x)=x$ as a spatially varying speed of light. Indeed, the geodesics equations come down to a simple $d x / d t= \pm x$ with a straightforward solution

$$
x(t)=x_{0} e^{ \pm t},
$$

with $x_{0}$ the initial position. When one switches to a proper time $\tau=x_{0} t / c$ of an observer at rest at $x=x_{0}$, then

$$
x(\tau)=x_{0} \exp \left( \pm c \tau / x_{0}\right) .
$$

The above result means that the light cones in the Rindler space-time are distorted and the strongest bending takes place in a vicinity of a horizon at $x=0$. Conversely, the bending of the light cone is negligible for $\tau / x_{0} \ll 1$, i.e., at very early times or far away from the horizon.

Because the information in the Rindler space-time does not propagate linearly, it is a central open question how the entanglement is propagating in such a setup. In the following, we argue the entanglement entropy in generic quantum systems in a Rindler space-time first grows sublinearly and asymptotically at long times attains a constant size-independent value in a thermodynamic limit.

\section{Setup}

In the following, we will consider the Hamiltonian description of free fermions in inhomogeneous space-times. As a first step, we investigate $(1+1)$ dimensional metric, but the generalization to higher dimensions is possible, and we plan to purse this path in the next future. Let us consider a system of spinless fermions on a one-dimensional (1D) lattice of length $N$ with open boundary conditions. We assume that an initial state is a spatially homogeneous product state

$$
\left|\Psi_{0}\right\rangle=\Pi_{n=1}^{N / 2} \hat{c}_{2 n}^{\dagger}|0\rangle,
$$

where $|0\rangle$ is a particle vacuum state, i.e., it is annihilated by any annihilation operator $\hat{c}_{n}|0\rangle=0$. Since the initial state is a product state, its entanglement entropy, Eq. (1), vanishes with respect to any bipartition. Suppose that at time $t=0$, we perform a quench such that the evolution of a system $t>0$ is described by a new Hamiltonian

$$
\hat{H}=-\frac{1}{2} \sum_{n=1}^{N} t_{n} \hat{c}_{n+1}^{\dagger} \hat{c}_{n}+\text { H.c. },
$$

where $c_{n}^{\dagger}, c_{n}$ are standard fermionic creation and annihilation operators on a lattice. It has been shown in Ref. [55] (see also Refs. [56-60]) that the massless Dirac fermions propagation on static space-times can be described by a lattice free fermion Hamiltonian with a spatially varying tunneling amplitude proportional to the determinant of the metric. For the noninteracting case, the spin degrees of freedom separate and therefore the Hamiltonian Eq. (7) corresponds to a spinless version of the discrete relativistic Hamiltonian on a curved $(1+1)$-dimensional space-time. Here, we choose $t_{n}=c=1$ 
or $t_{n}=n$. The first choice of tunneling amplitudes amounts to the Minkowski metric, Eq. (2), and the corresponding Hamiltonian is obviously a free fermionic Hamiltonian. From now on, we will refer to this choice of tunneling amplitudes as the homogeneous quench. The second choice of tunneling amplitudes $\left(t_{n}=n\right)$ entails the Rindler metric, Eq. (3), yielding the Rindler Hamiltonian

$$
\hat{H}=-\frac{1}{2} \sum_{n=1}^{N} n \hat{c}_{n+1}^{\dagger} \hat{c}_{n}+\text { H.c. }
$$

We note that this Hamiltonian, in a context of the BisognanoWichmann theorem, can be also interpreted as a modular or entanglement Hamiltonian. The study of entanglement Hamiltonian for lattice models has recently attracted substantial attention [61-69].

In this work, we investigate the entanglement evolution generated by the Rindler quench. In Sec. IV, we present the exact numerical diagonalization results for the entanglement entropy and in Sec. V we consider an analogous quench protocol in a unitary circuit setup. Before going to the main results, in Sec. II E, for illustrative and comparison purposes, we review the semiclassical pair quasiparticle picture for the homogeneous quench, which is well understood in the existing literature [18-33], and in Sec. III, we give its geometric interpretation in terms of propagation of correlations. On the contrary, because of the lack of the translational invariance in the Rindler Hamiltonian, Eq. (8), the (quasi)momenta are no longer good quantum numbers and the quasiparticle picture of counterpropagating pairs with opposite momenta does not hold. Nevertheless, since the speed of correlation spreading is still bounded in Sec. III we present heuristic arguments that allow us to extract important features of the entanglement dynamics, such as sublinear growth and a long-time asymptotically constant behavior. Most of all, we show that this approach allows us to identify relevant scaling parameters of the model.

\section{Entanglement entropy for free fermionic systems: the Peschel formula}

For the free fermionic models the entanglement entropy, between a subsystem $A$ and its complement $B$, can be efficiently calculated via correlation functions [70,71], as long as an initial state can be described by a Slater determinant. In this case, the density matrix can be written as an exponential of free fermionic operators

$$
\hat{\rho}_{A}=\frac{1}{Z} e^{-\hat{\mathcal{H}}_{A}}, \quad \hat{H}_{A}=\sum_{i, j \in A} h_{i j} \hat{c}_{i}^{\dagger} \hat{c}_{j},
$$

where $H=\left[h_{i j}\right]_{i, j \in A}$ matrix is diagonalized by the same transformation as a single particle correlation matrix $C=$ $\left[\left\langle\hat{c}_{i}^{\dagger} \hat{c}_{j}\right\rangle\right]_{i, j \in A}$. It has been found that the two matrices are related [70]

$$
H=\ln [(1-C) / C] .
$$

Using the thermal form of a reduced density matrix, Eq. (9), one can readily obtain an expression for the entanglement entropy following a quench at $t=0$

$$
S(t)=-\sum_{n=1}^{L}\left[\lambda_{n}(t) \ln \lambda_{n}(t)+\left[1-\lambda_{n}(t)\right] \ln \left[1-\lambda_{n}(t)\right]\right],
$$

where $\lambda_{n}(t)$ 's are eigenvalues of equal time correlation matrix

$$
C(t)=\left[\left\langle\hat{c}_{j}^{\dagger}(t) \hat{c}_{k}(t)\right\rangle\right]_{j, k \in A}
$$

restricted to a subsystem $A$. According to Eq. (11) the maximal value of the entanglement entropy $S_{\max }=L \ln 2$ is obtained if all $\lambda_{n}=1 / 2$, which corresponds to a trivial reduced density matrix, Eq. (9), i.e., an infinite temperature state of a subsystem.

Throughout this paper, we consider a bipartition $A$ and $B$, where $A=[m, m+L)$ is the smaller subsystem of length $L$ and $B$ is its complement, see Fig. 1(a). Also, by $\bar{m}=m+L / 2$, we denote the position of the middle of subsystem $A$, see Fig. 1(a).

\section{E. Quasiparticle geometric picture}

In the quasiparticle pair picture [18], an initial state $\left|\Psi_{0}\right\rangle$, being excited from the point of view of a quenched Hamiltonian $\hat{H}$, serves as a source of quasiparticle pair excitations. Each pair emitted from the same point in space is entangled and contribute to the total entanglement between $A$ and $B$, if at a time $t$ a pair is shared between the two regions. Accordingly, the entanglement entropy in the quasiparticle pair picture reads

$$
S(t)=2 t \int_{2 t|v(p)|<L} d p|v(p)| f(p)+L \int_{2 t|v(p)|>L} d p f(p),
$$

where $f(p)$ is a quasiparticle production rate and $v(p)=$ $d E(p) / d p$ is a quasiparticle velocity. If there exists a maximal quasiparticle velocity $v_{\max }$, than the entanglement entropy grows always linearly and saturates as a consequence of finite subsystem size at times $t \gtrsim t^{*}=L / 2$. The applicability of Eq. (13) goes beyond a simple qualitative understanding. In fact, in the thermodynamic limit $t, L \rightarrow \infty, t / L=$ const. the predictions of the quasiparticle picture exactly reproduce the behavior of entanglement entropy in 1D translationally invariant integrable models, based on the knowledge of the steady state and its excitations [28]. For arbitrary highly excited initial states, Eq. (13) describes the leading contribution to the entanglement entropy [31]. In the Appendix, using the quasiparticle formula, Eq. (13), we give an analytic form of the entanglement entropy for the staggered initial state and the homogeneous quench, Eq. (7). There, we show that the analytical formula is nonanalytical at $t^{*}=L / 2$, where the second derivative of entanglement entropy is discontinuous and we illustrate that even for relatively small $L$ 's the quasiparticle picture quite accurately reproduces the numerical data, although the nonanalyticity can only be observed in the infinite subsystem limit. In the Appendix, we also show that on contrary to the homogeneous case, where the entanglement entropy converges in a scaling limit $t, L \rightarrow \infty, t / L=$ const, the saturation effects of entanglement entropy after a Rindler quench are far from being universal and the same scaling 
(a)

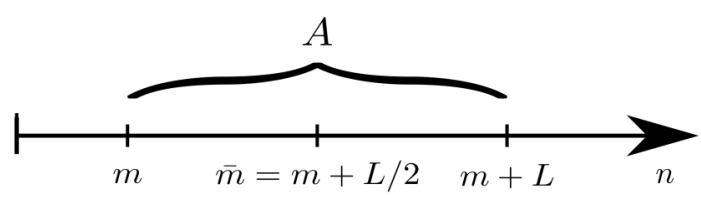

(b)

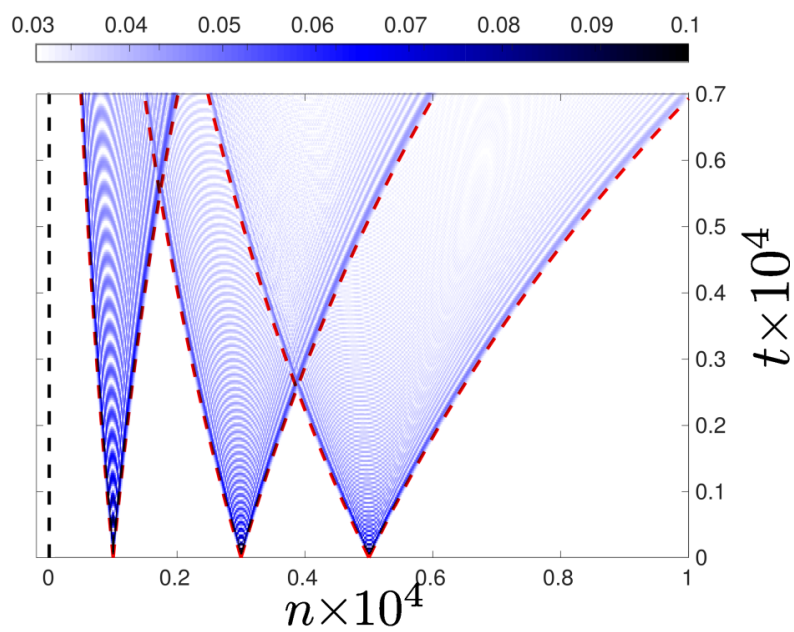

(C)

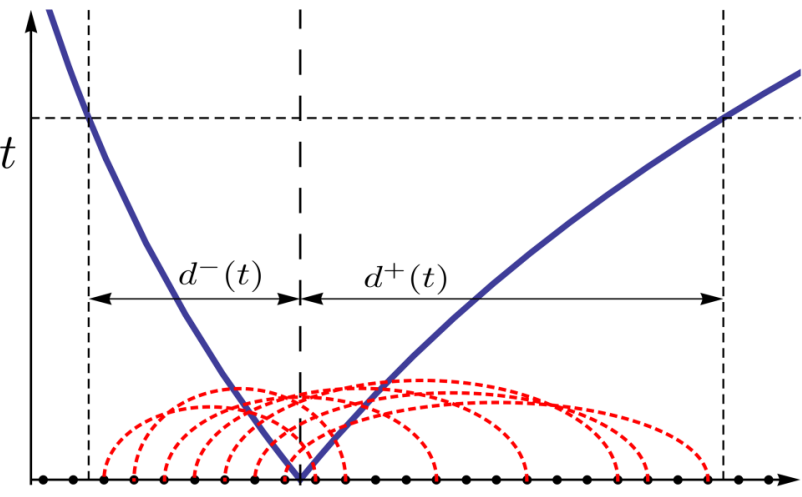

$m$

FIG. 1. (a) We assume a bipartition where a smaller subsystem $A=[m, m+L)$ is a segment with its mean position $\bar{m}=m+L / 2$. (b) The exact numerical lattice calculations of the correlation function $C_{n, n^{\prime}}(t)=\left\langle\hat{c}_{n}^{\dagger}(t) \hat{c}_{n^{\prime}}\right\rangle$, see Eq. (14), followed by a Rindler quench at $t=0$. We plot $C_{n, n^{\prime}}(t)$ as a function of $n$ and $t$ for different values of $n^{\prime}$. We observe a nonlinear spreading of correlations which form distorted light-cone structure. The light cone edges (red dashed lines) are described by a continuum theory, see Eq. (4). (c) Because of the light-cone bending, the number of lattice sites that are in a causal relation with a site $m$ depend on a spatial direction. We assume that the entanglement entropy is equal to the number of distinct pairs that can be correlated across the boundary between $A$ and $B$, i.e., it is proportional to $\min \left(d^{-}, d^{+}\right)=d^{-}$.

does not apply. For this reason, throughout the manuscript we focus on the entanglement entropy behavior up to times before saturation effects take place, i.e., for times smaller then the crossover time $t^{*}$.

\section{CORRELATION SPREADING}

The quasiparticle picture has a simple geometric interpretation. The fastest quasiparticles propagate at the maximum velocity $v_{\max }=\max _{k}|d E / d k|=1$, the Lieb-Robinson velocity [50], which is the maximal velocity of correlation spreading

$$
C_{n, n^{\prime}}(t)=\left\langle\hat{c}_{n}^{\dagger}(t) \hat{c}_{n^{\prime}}\right\rangle \equiv\left\langle\Psi_{0}\left|\hat{c}_{n}^{\dagger}(t) \hat{c}_{n^{\prime}}\right| \Psi_{0}\right\rangle,
$$

where $\left|\Psi_{0}\right\rangle$ is an initial state. In homogeneous systems, the correlations spread within linear light cones, which agrees with a quasiparticle pair picture. Geometrically, the contribution to the entanglement entropy $S(t)$ coming from each boundary between $A$ and $B$ is proportional to the length of an interval, $d(t)$, covered by a light cone placed at this boundary. Assuming that the entanglement entropy is proportional to the number of degrees of freedom which can become correlated at a time $t<t^{*}=L / 2$, the total entanglement entropy is then given by;

$$
S(t) \propto 2 d(t)=4 t .
$$

The quasiparticle picture of counterpropagating pairs with opposite quasimomenta cannot be applied directly if the postquench Hamiltonian does not possess translational invariance. However, similarly to the homogeneous quench, we can relate the entanglement entropy growth with the spreading of correlations. In Fig. 1(b), we plot the correlation function, Eq. (14) for a Rindler quench and for different choices of lattice positions $n^{\prime}$. The exact numerical calculations show that the correlations spread nonlinearly within bent light cones that are described by Eq. (4) with a very good agreement. In Fig. 1(c), we present a cartoon picture of a bent light cone originating at a lattice site $m$. Through a simple calculation a distance, $d^{-}(t)$ and $d^{+}(t)$, covered by a left and right part of the light cone accordingly is simply

$$
d^{ \pm}(t)=\mp m\left[1-e^{ \pm t}\right] .
$$

Now, we aim to apply semiclassical arguments to describe the entanglement dynamics. While this captures qualitative aspects of the entanglement dynamics, quantitative differences remain as we discuss in detail in the following. The direct application of a homogeneous result would lead to a superlinear growth of the entanglement entropy. Let us heuristically assume the entanglement entropy is proportional to the number of distinct pairs that can be correlated through each boundary between $A$ and $B$. This number is equal to $\min \left(d^{+}, d^{-}\right)=d^{-}$, see Fig. 1(c), and the total entanglement entropy is

$$
S(\tau) \propto 2\left[d_{1}^{-}(\tau)+d_{2}^{-}(\tau)\right]=4 \bar{m}\left[1-e^{-\tau / \bar{m}}\right],
$$

where $\bar{m}=(m+L / 2)$ is a position of the middle of $A$, and where $\tau=t \bar{m}$ is a proper time of an observer placed at $\bar{m}$. By switching to the proper time $\tau$, any stationary observer perceives the same local value of the speed of light $c(\bar{m}) / \bar{m}=$ 1 , which allows us to directly compare results with different observers' positions and different space-times.

The heuristic formula, Eq. (17), gives as an important insight into the behavior of entanglement entropy. First of all, it depends only on one parameter $\bar{m}$, and the dimensionless rescaled quantity $S(\tau \bar{m}) / \bar{m}$ is parameter-free. In Sec. IV, we find numerically the same scaling for free lattice fermions. Furthermore, the formula predicts universal sublinear growth growth of entanglement. Let us look at two opposing limits:

(1) if $\tau / \bar{m} \ll 1$, then $S(\tau) \propto 4 \tau$, so that locally and for early times a linear growth is recovered, 
(2) if $\tau / \bar{m} \gg 1$, then $S(\tau) \propto$ const.

Let us stress that the second limit is not the finite-size effect, but rather stems from general properties of the Rindler space-time where the horizon plays a role of an effective space-time boundary.

In the following sections, we show that the entanglement generated by the Rindler quench has both of the above properties. Nevertheless, we also find that the heuristic formula, Eq. (17), is too naive and does not recover quantitatively the numerical data. In fact, Eq. (17) considerably underestimate the entanglement entropy. One possible explanation is that the entanglement entropy requires important corrections from multiparticle entanglement, which is not captured in the above picture.

\section{NUMERICAL RESULTS}

In this section, we present numerical data for the entanglement entropy dynamics for the Rindler quench and stress the differences with the homogeneous case. We calculate the entanglement entropy by exact diagonalization of equal-time correlation matrices, see Sec. IID. In Sec. IV A, we show that the entanglement growth features universal behavior by achieving a data collapse, while in Sec. IV B, we discuss finite-size effects in more details. Surprisingly, the finite-size analysis allows us to identify a universal long-time asymptotic behavior.

\section{A. Universal sublinear growth}

In the case of the homogeneous quench, the entanglement entropy depends only on the subsystem size $L$ and the generic behavior can be numerically extracted by overlaping data for different subsystem sizes $L$. For the Rindler quench, due to the inhomogeneous metric, we find that the entanglement entropy depends also on the mean position of the subsystem $A$, see the semiclassical discussion in Sec. III. Therefore, in order to extract the universal behavior from the numerics, we need to fix a mean position $\bar{m}$ first and then perform the finite data overlap. In Fig. 2, the entanglement entropy is plotted versus a proper time $\tau=t \bar{m}$ of observer located at $\bar{m}$. For the sake of comparison, we include data for both the Rindler (solid lines) and homogeneous (dashed lines) quenches.

At early times and small subsystem sizes $L$ the data for Rindler and homogeneous quenches are indistinguishable [this is expected as initial light cone bending from the point of view of local observers is small, see Eq. (5)]. The inhomogeneity of the space-time becomes important at timescales $\tau \approx \bar{m}$, where the entanglement entropy for the Rindler quench exhibits sublinear growth behavior. For both quenches, we observe the collapse of the data and a generic entanglement function $S_{\bar{m}}(\tau)$ can be identified as an envelope of a family of curves, $S_{\bar{m}, L}(\tau)$, with fixed $\bar{m}$ and different $L$. In other words, $S_{\bar{m}}(\tau)$ can be defined a limit $S_{\bar{m}}(\tau)=$ $\lim _{L \rightarrow 2 \bar{m}} S_{\bar{m}, L}(\tau)$.

Let us now rescale the numerical curves $S_{\bar{m}}(\tau)$ for different values of $\bar{m}$

$$
S_{\bar{m}}\left(\tau^{\prime}\right)=S_{\bar{m}}\left(\bar{m} \tau^{\prime}\right) / \bar{m} .
$$

According to the semiclassical formula, Eq. (17), the $s_{\bar{m}}\left(\tau^{\prime}\right)$ curves should overlap. Indeed, although small
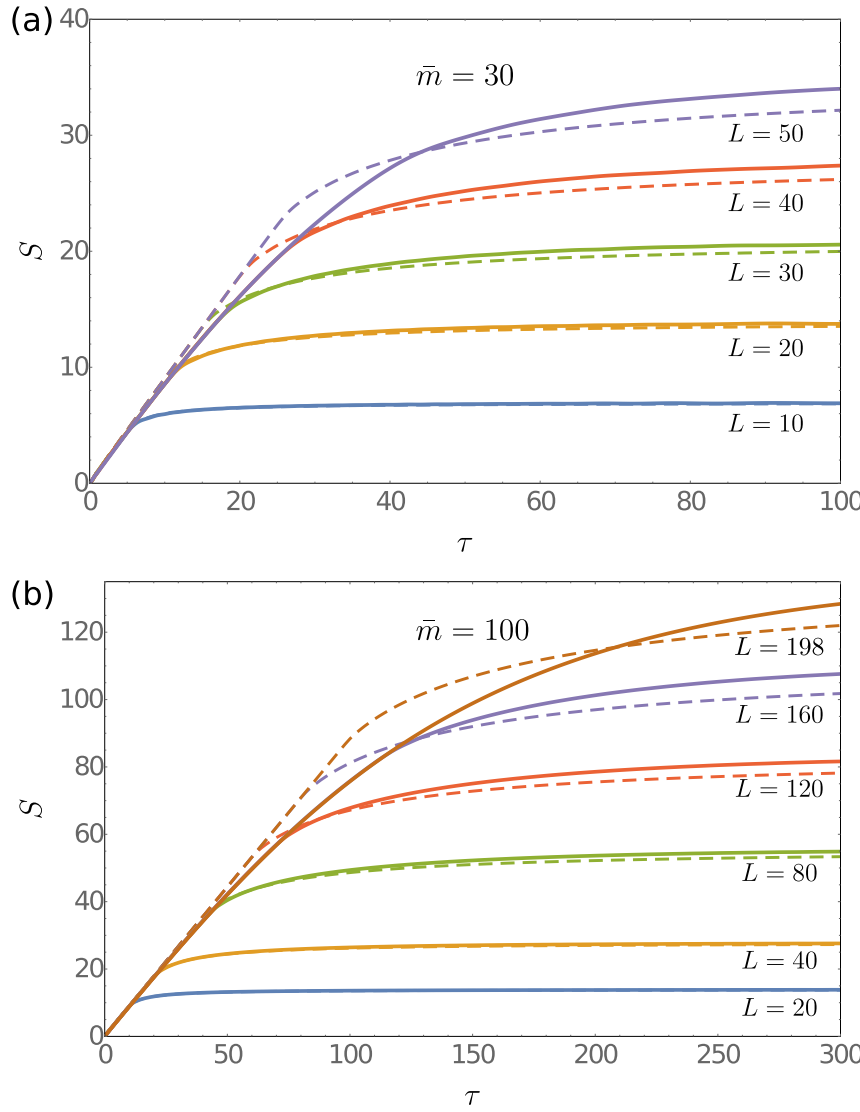

FIG. 2. The entanglement entropy obtained from the numerical computations. In order to extract a generic behavior, we fix the mean position of the subsystem and perform finite size overlap of the data. Here we choose $\bar{m}=30$ (a) and 100 (b). For comparison, we include data for both the Rindler (solid lines) and homogeneous quench (dashed lines). $\tau$ denotes a proper time of an observer, see discussion in the main text. Apart from the finite subsystem size saturation to the $S_{\max }=L \ln 2$, we find that all curves for different subsizes $L$ 's and fixed mean positions $\bar{m}$ overlap, which allows us to identify a generic functional behavior. The numerical data confirm that $\bar{m}$ is the only relevant parameter and that the entanglement entropy grows sublinearly. Furthermore, we observe peculiar finite-size features of the Rindler quench: although the entanglement entropy grows slower then in the homogeneous case, the entanglement entropy saturates much faster.

deviations are found for small $\bar{m}$ 's, in the limit $1 / \bar{m} \rightarrow 0$, we observe a convergence of the data, i.e., $s\left(\tau^{\prime}\right)$ defined as

$$
s\left(\tau^{\prime}\right)=\lim _{1 / \bar{m} \rightarrow 0} s_{\bar{m}}\left(\tau^{\prime}\right)
$$

has a thermodynamic limit, see Fig. 3. This suggests a universal sublinear behavior of the entanglement entropy growth. On the contrary to the homogeneous case, the entropy production rate (the first derivative of entanglement entropy) decreases over time and, as we argue in the next section, the entropy is asymptotically constant in a long-time limit.

\section{B. Asymptotic long-time dynamics}

In this section, we analyze finite subsystem size effects in entanglement entropy evolution, which allows us to identify 


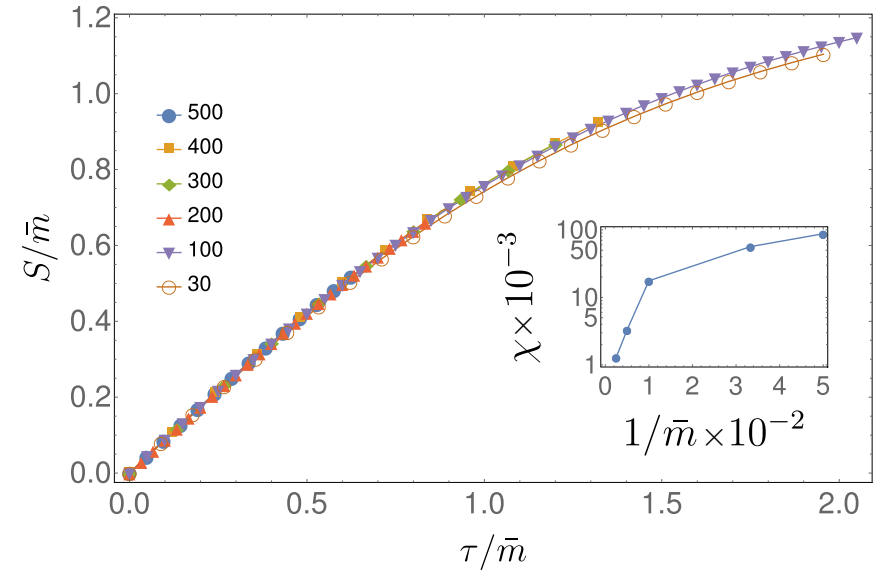

FIG. 3. Comparison of the entanglement entropy curves in the case of the Rindler quench, obtained from the finite-size data collapse for a fixed $\bar{m}=30, \ldots, 500$. All curves are rescaled accordingly to $s_{\bar{m}}\left(\tau^{\prime}\right)=S_{\bar{m}}\left(\bar{m} \tau^{\prime}\right) / \bar{m}$. The inset shows $\chi_{\bar{m}^{\prime}}=\left[\int \mathrm{d} \tau\left(s_{\bar{m}=500}(\tau)-\right.\right.$ $\left.\left.s_{\bar{m}^{\prime}}(\tau)\right)^{2}\right]^{1 / 2}$, which is the measure of distance between two functions. In the limit $1 / \bar{m} \rightarrow 0$, we observe a converge of data.

universal asymptotic long-time dynamics in the thermodynamic limit. Already in Fig. 2 one could notice two striking saturation effect differences between the Rindler and homogeneous quenches. First of all, a crossover time $\tau^{*}$-a time when the evolution starts to thermalize due to a finite size of a subsystem-increases together the growth of a subsystem size $L$. Secondly, the finite size thermalization is much faster.

Let us first quantify the first observation. We recall that $\bar{m}$ denotes a middle position of a subsystem $A$ of length $L$, and therefore the $L / \bar{m} \in[1 / \bar{m}, 2-2 / \bar{m}]$. The lower limit corresponds to a situation when $A$ of a unit length is far away from the origin $m=1$, while the upper limit corresponds to $A$ located maximally close to the origin. Previously, we have identified a generic function $S_{\bar{m}}(\tau)$ which is an envelope of a family of curves $S_{\bar{m}, L}(\tau)$ with fixed $L$. We define a crossover time $\tau^{*}(L)$ as $\left|S_{\bar{m}, L}(\tau)-S_{\bar{m}}(\tau)\right|<\epsilon$ and in Fig. 4, we plot $\tau^{*} / L$ as function of $L / \bar{m}$ for $\bar{m}=200$ and $\epsilon=0.03$. While the choice for the threshold $\epsilon$ is, of course, arbitrary, we find that the final result for $\tau^{*}(L)$ doesn't depend crucially on it as long as $\epsilon$ is sufficiently small. As expected, we observe that $\tau^{*} \approx L / 2$ when the subsystem $A$ is small comparing to its distance to the origin, which recovers the known homogeneous result. This is perfectly understandable, since for small $\mathrm{L} / \mathrm{m}$ the effective speed of light $c(m) \propto m$ is locally constant and does not change significantly on the extent of a subsystem, i.e.,

$$
c(m+L / 2) / c(m-L / 2) \approx 1+L / m .
$$

On the other hand, $\tau^{*}$ increases drastically when $L / \bar{m}$ ratio approaches its maximal value $2-2 / \bar{m}$ and in the thermodynamic limit $\bar{m} \rightarrow \infty$, the function $\tau^{*} / L$ becomes infinite at $L / \bar{m}=2$, see Fig. 4. From this seemingly small result, we can actually infer the asymptotic long time behavior of the universal curve $s(\tau)$, Eq. (19). Since $\forall_{\tau} S_{\bar{m}, L}(\tau) \leqslant L \ln 2$ and $S_{\bar{m}}(\tau)=\lim _{L \rightarrow 2 \bar{m}} S_{\bar{m}, L}(\tau)$, then

$$
\lim _{L \rightarrow 2 \bar{m}} S_{\bar{m}, L}\left(\tau^{*}\right)=S_{\bar{m}}(\infty)=2 \bar{m} \ln 2
$$

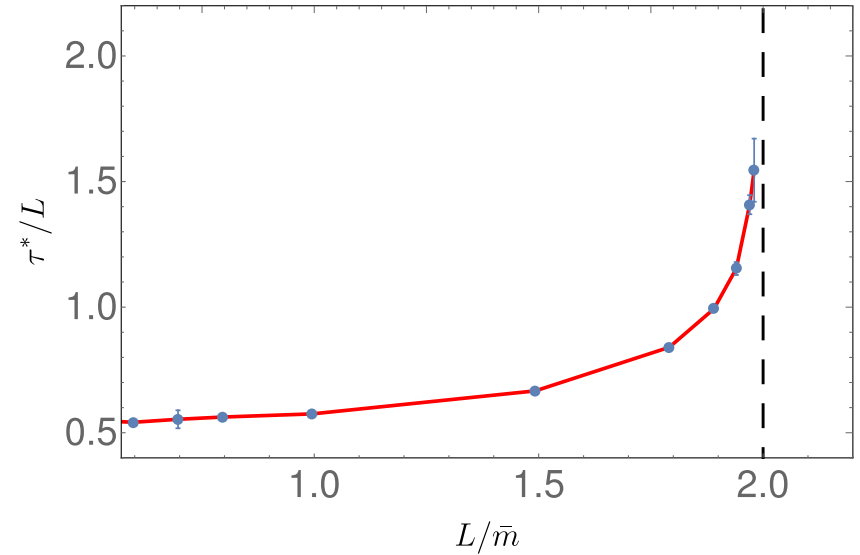

FIG. 4. Crossover times $\tau^{*}$ vs the ratio $L / \bar{m}$ obtained from numerical data. We definite a crossover time $\tau^{*}$ of the entanglement entropy growth when the finite subsystem size saturation effects start to take place. Initially, for small ratios $L / \bar{m} \lesssim 1$, the critical time is $\tau^{*} \approx L / 2$, which agrees with the result from the homogeneous quench. The critical time grows drastically when the ratio ratio $L / \bar{m}$ increases and approaches its maximally attainable value equal to $2-2 / \bar{m} . \tau^{*} / L$ becomes infinite in the limit $\bar{m} \rightarrow \infty$ such that $L / \bar{m} \rightarrow 2$.

and consequently $s(\tau)$ has a horizontal asymptote

$$
\lim _{\tau \rightarrow \infty} s(\tau)=2 \ln 2
$$

Let us stress that although the functional behavior of the entanglement entropy might be different for different initial states, the limit in Eq. (22) is generic for a wide class of highly excited homogeneous initial states. In physical terms, the thermodynamic limit $\bar{m} \rightarrow \infty$ while $L / \bar{m} \rightarrow 2$ means that one edge of the subsystem $A$ is placed basically at the horizon, where the local light velocity vanishes asymptotically. As a consequence, it would require an infinite time for the signal to propagate throughout the subsystem and the crossover time $\tau^{*}$ has to diverge accordingly. Note that this behavior was already predicted on a semiclassical level, Sec. III.

Finally, we investigate the entanglement entropy saturation rate, i.e., how fast the subsystem thermalizes at times scales much larger than the crossover time. In Fig. 5, we plot the distance $\left|S(\tau)-S_{\max }\right|$ to the maximal value $S_{\max }=L \ln 2$ in time. It is known that in a case of global quench the entanglement entropy saturates as $L^{2} / t$ [18] (see also Appendix). On the other hand, in a case of a Rindler quench, we can see that after times $\tau \approx \bar{m}$ a subsystem thermalizes exponentially fast, which we find very intriguing: although the entanglement entropy grows sublinearly, it saturates much faster than in a homogeneous case. Last but not least let us stress that the saturation effects of the entanglement entropy for a Rindler quench are in general not universal and the scaling limit $t, L \rightarrow \infty, t / L=$ const is a proper one only when the crossover time diverges. In this case, we can quantify the numerical results by fitting the prediction from the unitary circuits analysis, see Sec. V. There, we obtain that $S(t) \propto \tanh ($ const. $t / \bar{m})=\tanh (2$ const. $t / l)$. 


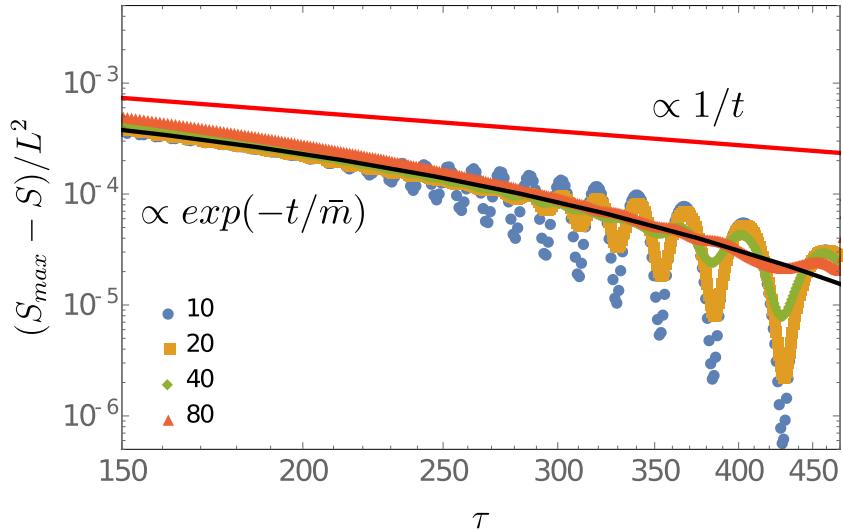

FIG. 5. The distance to the asymptotic maximal value of entanglement entropy $\left|S(\tau)-S_{\max }\right|$ vs time for a fixed value $\bar{m}=100$. Although initially the entanglement entropy for a Rindler quench grows sublinearly, we find that it approaches the thermodynamic value $S_{\max }=L \ln 2$ exponentially fast, while for a global quench the entanglement entropy (red curve) saturates as $S \propto L^{2} / t$.

\section{RANDOM UNITARY CIRCUITS}

In the previous sections, we have investigated the entanglement entropy evolution after the Rindler quench in a simple system of free fermions on a lattice. In particular, we have found that a simple heuristic semiclassical arguments (Sec. III) give us important insight into the universal behavior of entanglement dynamics, although it fails to quantitatively reproduce the numerical data (Sec. IV). The semiclassical arguments cannot account for multiparticle contribution to the entanglement, which is most probably the reason of quantitatively differences. On the other hand, such mutliparticle contribution can be captured in random unitary circuit setups, which provide minimally structured toy models of generic nonintegrable systems. For this reason, in this part, we estimate the entanglement entropy for a specific system of random unitary circuits, where the coarse grained entanglement dynamics is equivalent to the minimal membrane description [45].

Let us denote $S(x, t)$ for the entanglement entropy for an arbitrary quantum state, where $x$ is a position of a bipartite cut. If, like in the previous section, we would rather consider a bipartition where a subsystem $A$ is a finite segment $A=$ $\left[m_{1}, m_{2}\right]$, then the entanglement entropy of such a bipartition is $S_{A}(t)=S\left(m_{1}, t\right)+S\left(m_{2}, t\right)$. After Ref. [45], we write down the equation for the leading order coarse-grained dynamics of the local rate of the entanglement entropy

$$
\partial_{t} S(x, t)=\Gamma\left[s=\partial_{x} S(x, t)\right],
$$

where $\Gamma(s)$ is a production rate

$$
\Gamma(s)=\gamma\left(1-\alpha s^{2}\right),
$$

where $\alpha, \gamma$ are free parameters. While Eq. (23) is universal for a generic random unitary circuit setup, a specific model can be achieved by fixing free parameters $\alpha$ and $\gamma$, see Ref. [45]. Let us note that in general the above formula should be treated as Taylor expansion of the entanglement line tension, where the higher order terms do not contribute to the coarse grained dynamics as long as $\partial_{x} S(x, t)$ is sufficiently small.

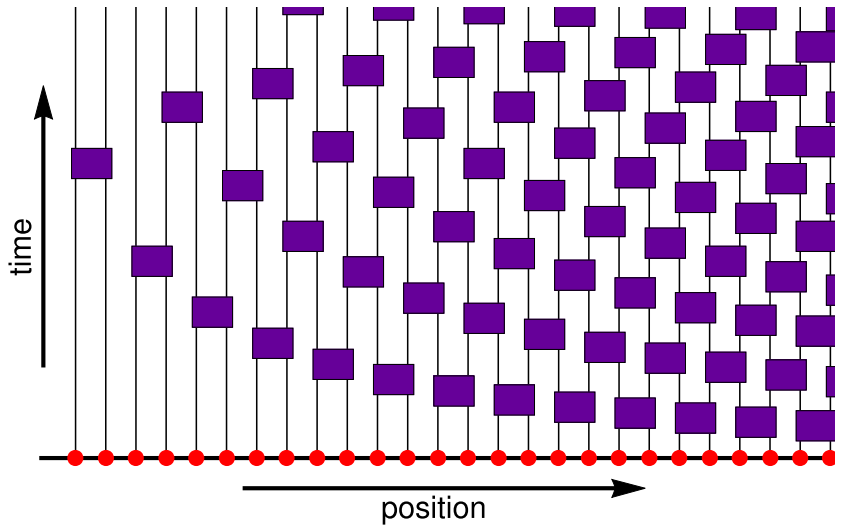

FIG. 6. A schematic structure for simulating a random unitary circuits with a Rindler metric. Red points represents an initial spin configuration and purple rectangles gates are random unitary gates. The rate at which random unitary gates are applied is an inverse function of a distance to the horizon placed at the left boundary of the system.

Our goal is to describe the entanglement entropy dynamics in a Rindler quench scenario. To make it work, we simply notice that in Rindler Universe, Eq. (3), due to spatially varying speed of light $c(x)=x$, the local dynamics is the slower the closer are to $x=0$, and so $\gamma=\beta x$ should be proportional to $x$, yielding

$$
\partial_{t} S(x, t)=x \beta\left[1-\alpha\left(\partial_{x} S(x, t)\right)^{2}\right] .
$$

In a same way, to simulate a random circuits with a Rindler metric one should apply random unitary gates with rates proportional to the inverse distance to the horizon, see Fig. 6 for an illustration. Now, by utilizing an ansatz for separation of variables

$$
S(x, t)=x f(t)
$$

it is straightforward to solve the corresponding differential equation

$$
\partial_{t} f(t)=\beta\left(1-\alpha f(t)^{2}\right),
$$

which has an elementary solution

$$
f(t)=\frac{1}{\sqrt{\alpha}} \tanh (\sqrt{\alpha} \beta t) .
$$

Finally, then the entanglement entropy between $A$ and its complement reads

$$
S_{A}(\tau)=\frac{2 \bar{m}}{\sqrt{\alpha}} \tanh (\sqrt{\alpha} \beta \tau / \bar{m}),
$$

where $\bar{m}=\left(m_{1}+m_{2}\right) / 2$ and $\tau=\bar{m} t$ is a proper time of an observer located at $\bar{m}$.

As we have obtained from the semiclassical analysis, where $S(\tau)=\bar{m}\left(1-e^{-\tau / \bar{m}}\right)$, see Sec. III, the entanglement entropy growth predicted by the random unitary circuit, Eq. (29), is linear at early times and attains a constant values at long times scales. On the contrary, we know that the entanglement entropy of random unitary circuit models could in principle capture multiparticle contribution to the entanglement. Therefore, although the two formulas obey the same 


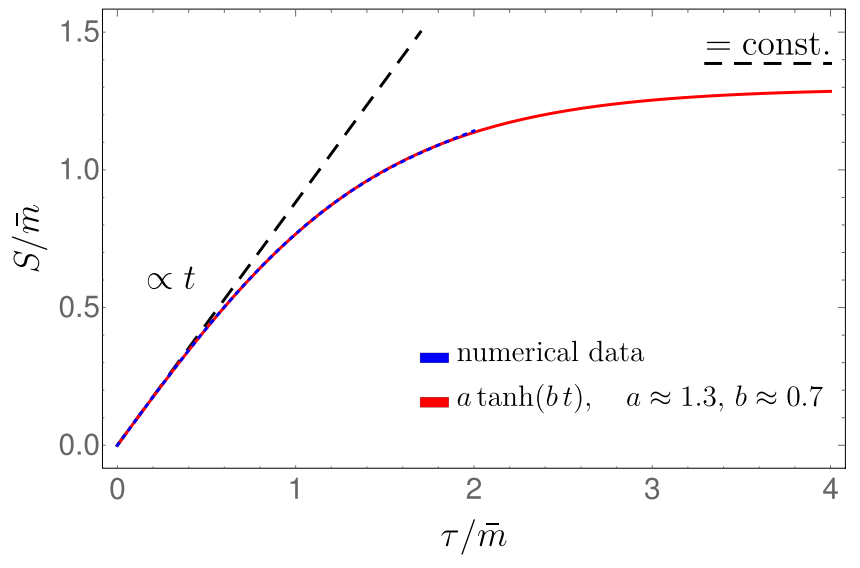

FIG. 7. Comparison of the entanglement entropies for a Rindler quench and for a free fermionic chain obtained from exact numerical data (blue dashed) and from the random unitary circuits model (red).

scaling $s(\tau)=S(\tau \bar{m}) / \bar{m}$ and are qualitatively similar, we can expect quantitative differences both in the short and long time behavior. In particular, at short time scales the first non vanishing correction to the linear growth in $S(\tau)$ is quadratic while in $S_{A}(\tau)$ the first correction is cubic. At long time scales it is straightforward to find that $S_{A}(\tau) \propto 1-2 e^{-2 \sqrt{\alpha} \beta \tau / \bar{m}}$.

Notice that the entanglement entropy $S_{A}(\tau)$, Eq. (29), depends on two free parameters which correspond to different choices of the entropy production rate. Since $\alpha, \beta$ are two unknown model specific parameters, $S_{A}(\tau)$ should also recover the numerical results obtained for noninteracting fermions. Let us therefore fit $s_{A}(\tau)=S_{A}(\tau \bar{m}) / \bar{m}$ to the numerical data from the Sec. IV. We plot the results in Fig. 7 and find that the curve remarkably agrees with the numerical data with fitting coefficients $\alpha \approx 2.4, \beta \approx 0.45$. This suggests that our result captures universal properties of entanglement entropy dynamics after the Rindler quench.

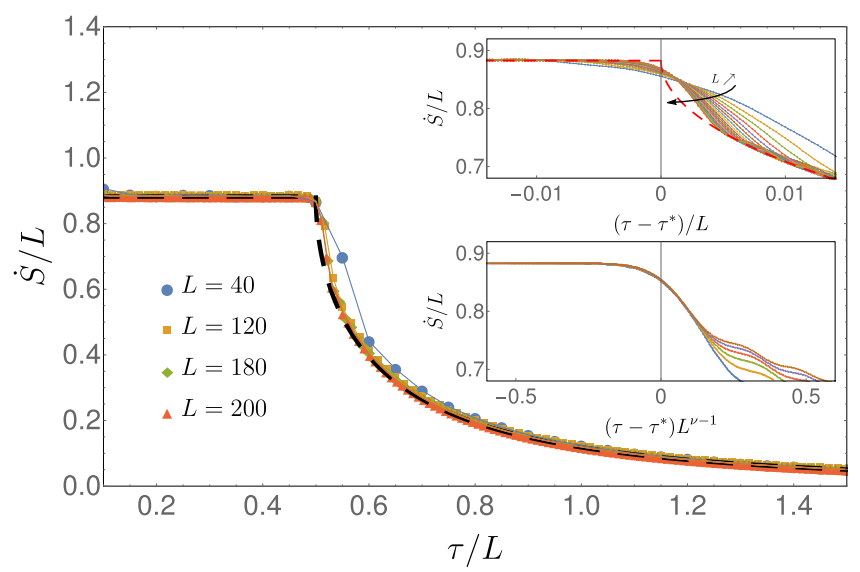

FIG. 8. The Entanglement entropy after a global quench. The analytical prediction for the entanglement entropy $\dot{s}(t)$ (black dashed line) and the exact numerical data for finite subsystem sizes. The upper inset shows that increasing the subsystem size (up to $L=$ $2 \times 10^{4}$ ), the numerical data get closer to the analytical prediction. The lower inset illustrates a collapse of data after a finite-size scaling. The scaling coefficient is $v \approx 0.5$.
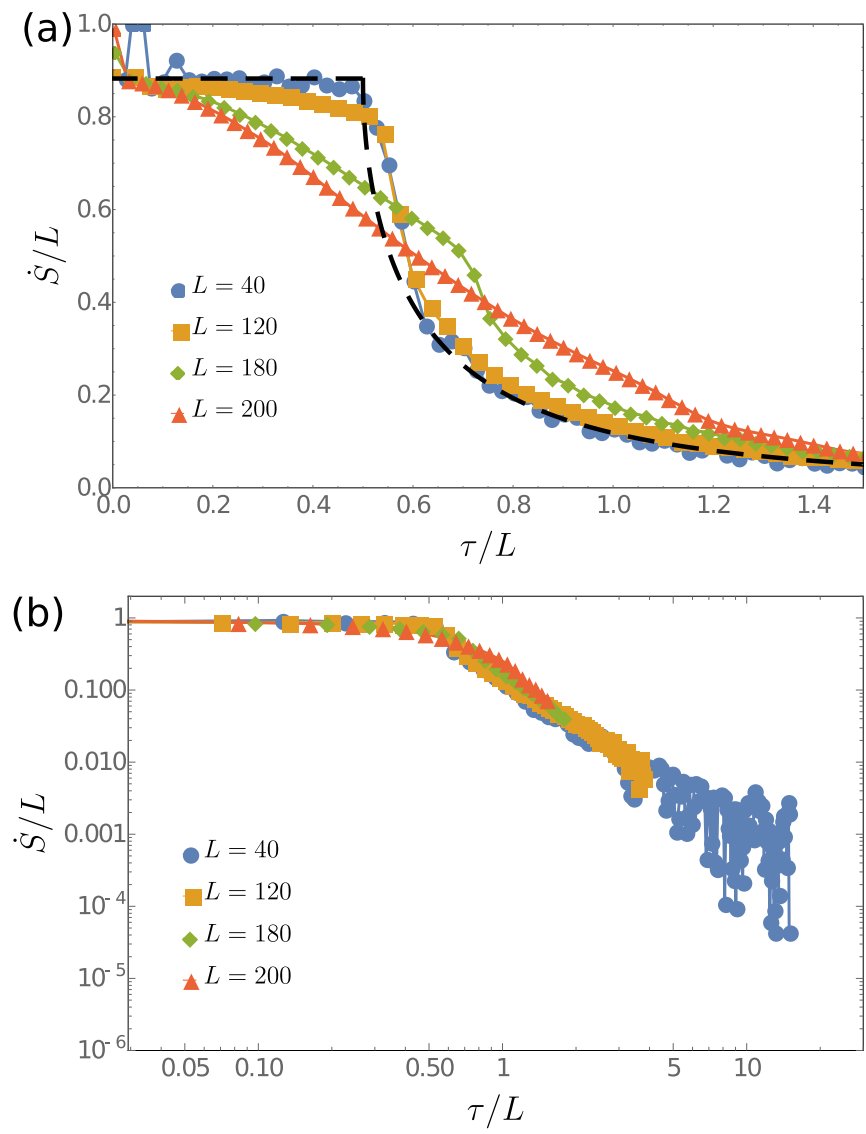

FIG. 9. The entanglement entropy after a Rindler quench. The numerical derivative of the entanglement entropy. The black dashed line is an analytic line $\dot{s}(t)$ obtained for a global quench. We find that with the increasing $L$ the derivative becomes smoother around $t^{*}=L / 2$. (a) and (b) shows the same data on linear and logarithmic scales accordingly. The computations were performed for $\bar{m}=200$.

\section{CONCLUSIONS}

The main goal of this work was to study entanglement growth in inhomogeneous space-times, where correlations do not propagate within straight light cones. We have taken as an example the $(1+1)$ Rindler metric, which is known to have a space-time horizon that strongly distorts the light cones in its vicinity. We have shown that the entanglement initially grows sublinearly and in a long-time limit is asymptotically constant. This behavior can be qualitatively understood via semiclassical arguments that base on the knowledge of correlation spreading. Correlations spread within distorted light cones that are described by null geodesics of the Rindler space-time. This suggests that we have captured basic properties of the continuum theory and that the similar reasoning can be applied to other space-times.

We have found indications that our observations are universal. For the paradigmatic example of an ergodic system, we have studied the entanglement growth also for a random unitary circuit model. Choosing a specific random unitary circuit setup, we have derived a leading coarse-grained dynamics of the entanglement entropy that has the same characteristics as in a case of free fermions on the lattice. This suggests that our results are applicable to a generic quantum system. This 
opens the way towards studying entanglement production in more general inhomogeneous space-times.

\section{ACKNOWLEDGMENTS}

We acknowledge a very fruitful discussion with Adam Nahum. A.K. would like to thank Alessio Celi, Maciej Lewenstein, and Emanuele Tirrito for previous discussions concerning Rindler physics. A.K. acknowledge the support of the Foundation for Polish Science (FNP) and the support of the National Science Centre, Poland via Projects No. 2016/21/B/ST2/01086 (A.K.). This project has received funding from the European Research Council (ERC) under the European Union's Horizon 2020 research and innovation programme (Grant Agreement No. 853443), and M.H. further acknowledges support by the Deutsche Forschungsgemeinschaft (DFG) via the Gottfried Wilhelm Leibniz Prize program.

\section{APPENDIX: NONANALYTIC BEHAVIOR OF THE ENTANGLEMENT ENTROPY}

The entanglement entropy due to quasiparticle picture [18] reads

$$
S(t)=2 t \int_{2 t|v(p)|<L} d p|v(p)| f(p)+L \int_{2 t|v(p)|>L} d p f(p),
$$

where $f(p)$ is a quasiparticle production rate, that we obtain from the properties of the stationary state [31], and $v(p)=$ $d E(p) / d p=\cos (p)$ is a simple lattice dispersion relation for free particles. For a global homogeneous quench that we consider the main part of the paper, we obtain

$$
S(t)=\left\{\begin{array}{lc}
S_{\text {lin }}(t) & , \quad 0 \leqslant t \leqslant L / 2 \\
S_{\mathrm{sat}}(t) & , \quad t>L / 2
\end{array},\right.
$$

where

$$
\begin{gathered}
S_{\operatorname{lin}}(t)=\frac{4 \ln 2}{\pi} t, \\
S_{\text {sat }}(t)=\frac{2 L \ln 2}{\pi}\left[\arccos \left(\frac{L}{2 t}\right)+\frac{2 t-\sqrt{4 t^{2}-L^{2}}}{L}\right] .
\end{gathered}
$$

From general considerations, Calabrese and Cardy [18] argue that at late times $t \ll L / 2$ the entanglement entropy $S(t)$ saturates as $\propto L^{2} / t$. In our specific case, we can calculate the asymptotic behavior exactly. The Taylor expansion of $S_{\text {sat }}(t)$ at small $x=L / 2 t$ yields

$$
S_{\text {sat }}(t \ll L / 2)=L \ln 2-\frac{L^{2} \ln 2}{2 \pi t}+o\left((L / 2 t)^{2}\right) .
$$

It is convenient to rescale $s(t) \equiv S(t L) / L$, which does not depend on $L$, such that the thermodynamic limit $t, L \rightarrow \infty$, but $t / L=$ const. is straightforward. The function $s(t)$ belongs to the $\mathcal{C}^{1}$ differentiability class, i.e., its derivative is not differentiable at $t=t^{*}$. We plot $s(t)$ together with the numerical data in Fig. 8. We see that even for relatively small $L$ 's the numerical data reproduces the analytic curve almost perfectly, where the divergence can be observed in a vicinity of $t^{*}$. Yet, the finite-size analysis confirms non analytical behavior of $s(t)$ in the thermodynamic limit, see insets of Fig. 8.

In the main text of this paper, we have shown that the saturation effects of entanglement entropy after a Rindler quench are far from being universal. In Fig. 9, as before, we plot the first derivative of the entanglement entropy for different subsystem sizes $L$ and fixed $\bar{m}=200$, see Fig. 8 for a comparison. The derivative gets smoother with increasing $L$ and the crossover point becomes less pronounced.
[1] I. Bengtsson and K. Zyczkowski, Geometry of Quantum States: An Introduction to Quantum Entanglement (Cambridge University Press, Cambridge, UK, 2006).

[2] R. Horodecki, P. Horodecki, M. Horodecki, and K. Horodecki, Rev. Mod. Phys. 81, 865 (2009).

[3] A. K. Ekert, Phys. Rev. Lett. 67, 661 (1991).

[4] N. Gisin, G. Ribordy, W. Tittel, and H. Zbinden, Rev. Mod. Phys. 74, 145 (2002).

[5] S. Pirandola, U. L. Andersen, L. Banchi, M. Berta, D. Bunandar, R. Colbeck, D. Englund, T. Gehring, C. Lupo, C. Ottaviani et al., Advances in quantum cryptography, arXiv:1906.01645 (2019).

[6] C. H. Bennett, G. Brassard, C. Crépeau, R. Jozsa, A. Peres, and W. K. Wootters, Phys. Rev. Lett. 70, 1895 (1993).

[7] S. Pirandola, J. Eisert, C. Weedbrook, A. Furusawa, and S. L. Braunstein, Nat. Photonics 9, 641 (2015).

[8] R. P. Feynman, Int. J. Theor. Phys. 21, 467 (1982).

[9] A. Steane, Rep. Prog. Phys. 61, 117 (1998).

[10] T. D. Ladd, F. Jelezko, R. Laflamme, Y. Nakamura, C. Monroe, and J. L. OBrien, Nature 464, 45 (2010).

[11] P. Hauke, F. M. Cucchietti, L. Tagliacozzo, I. Deutsch, and M. Lewenstein, Rep. Prog. Phys. 75, 082401 (2012).
[12] I. M. Georgescu, S. Ashhab, and F. Nori, Rev. Mod. Phys. 86, 153 (2014).

[13] L. Amico, R. Fazio, A. Osterloh, and V. Vedral, Rev. Mod. Phys. 80, 517 (2008).

[14] N. Laflorencie, Phys. Rep. 646, 1 (2016).

[15] C. Holzhey, F. Larsen, and F. Wilczek, Nucl. Phys. B 424, 443 (1994).

[16] P. Calabrese and J. Cardy, J. Stat. Mech. (2004) P06002.

[17] J. Eisert, M. Cramer, and M. B. Plenio, Rev. Mod. Phys. 82, 277 (2010).

[18] P. Calabrese and J. Cardy, J. Stat. Mech. (2005) P04010.

[19] M. Fagotti and P. Calabrese, Phys. Rev. A 78, 010306(R) (2008).

[20] M. G. Nezhadhaghighi and M. A. Rajabpour, Phys. Rev. B 90 , 205438 (2014).

[21] L. Bucciantini, M. Kormos, and P. Calabrese, J. Phys. A: Math. Theor. 47, 175002 (2014).

[22] J. Cardy and E. Tonni, J. Stat. Mech. (2016) 123103.

[23] J. S. Cotler, M. P. Hertzberg, M. Mezei, and M. T. Mueller, J. High Energy Phys. 11 (2016) 166.

[24] X. Wen, S. Ryu, and A. W. W. Ludwig, J. Stat. Mech. (2018) 113103. 
[25] K. Najafi, M. A. Rajabpour, and J. Viti, Phys. Rev. B 97, 205103 (2018).

[26] H. Kim and D. A. Huse, Phys. Rev. Lett. 111, 127205 (2013).

[27] A. S. Buyskikh, M. Fagotti, J. Schachenmayer, F. Essler, and A. J. Daley, Phys. Rev. A 93, 053620 (2016).

[28] V. Alba and P. Calabrese, Proc. Natl. Acad. Sci. U.S.A. 114, 7947 (2017).

[29] V. Alba and P. Calabrese, SciPost Phys. 4, 17 (2018).

[30] V. Alba, Phys. Rev. B 97, 245135 (2018).

[31] B. Bertini, E. Tartaglia, and P. Calabrese, J. Stat. Mech. (2018) 063104.

[32] J. Viti, J.-M. Stéphan, J. Dubail, and M. Haque, Europhys. Lett. 115, 40011 (2016).

[33] J. Dubail, J.-M. Stéphan, J. Viti, and P. Calabrese, SciPost Phys. 2, 002 (2017).

[34] B. Bertini, M. Fagotti, L. Piroli, and P. Calabrese, J. Phys. A: Math. Theor. 51, 39LT01 (2018).

[35] V. Alba, B. Bertini, and M. Fagotti, SciPost Phys. 7, 005 (2019).

[36] J. H. Bardarson, F. Pollmann, and J. E. Moore, Phys. Rev. Lett. 109, 017202 (2012).

[37] M. Serbyn, Z. Papić, and D. A. Abanin, Phys. Rev. Lett. 110, 260601 (2013).

[38] D. A. Huse, R. Nandkishore, and V. Oganesyan, Phys. Rev. B 90, 174202 (2014).

[39] M. Schreiber, S. S. Hodgman, P. Bordia, H. P. Lüschen, M. H. Fischer, R. Vosk, E. Altman, U. Schneider, and I. Bloch, Science 349, 842 (2015).

[40] D. A. Abanin, E. Altman, I. Bloch, and M. Serbyn, Rev. Mod. Phys. 91, 021001 (2019).

[41] N. D. Birrell and P. C. W. Davies, Quantum Fields in Curved Space, Cambridge Monographs on Mathematical Physics (Cambridge University Press, Cambridge, UK, 1982).

[42] S. Takagi, Prog. Theor. Phys. Suppl. 88, 1 (1986).

[43] R. M. Wald, General Relativity (University of Chicago press, Chicago, Illinois, 2010).

[44] A. Nahum, J. Ruhman, S. Vijay, and J. Haah, Phys. Rev. X 7, 031016 (2017).

[45] C. Jonay, D. A. Huse, and A. Nahum, Coarse-grained dynamics of operator and state entanglement, arXiv:1803.00089.

[46] A. Nahum, S. Vijay, and J. Haah, Phys. Rev. X 8, 021014 (2018).

[47] A. Nahum, J. Ruhman, and D. A. Huse, Phys. Rev. B 98, 035118 (2018).
[48] M. B. Plenio and S. S. Virmani, in Quantum Information and Coherence (Springer, Cham, Switzerland, 2014), pp. 173-209.

[49] S. Maity, S. Bandyopadhyay, S. Bhattacharjee, and A. Dutta, Phys. Rev. B 101, 180301(R) (2020).

[50] E. H. Lieb and D. W. Robinson, in Statistical Mechanics (Springer, Berlin, Heidelberg, 1972), pp. 425-431.

[51] X. Wen and J.-Q. Wu, Floquet conformal field theory, arXiv: 1805.00031.

[52] B. Lapierre, K. Choo, C. Tauber, A. Tiwari, T. Neupert, and R. Chitra, Phys. Rev. Research 2, 023085 (2020).

[53] K. Schwarzschild, Sitzungsber. Preuss. Akad. Wiss. Berlin (Math. Phys.) 1916, 424 (1916).

[54] W. G. Unruh, Phys. Rev. D 14, 870 (1976).

[55] O. Boada, A. Celi, J. I. Latorre, and M. Lewenstein, New J. Phys. 13, 035002 (2011).

[56] A. Celi, Eur. Phys. J.: Spec. Top. 226, 2729 (2017).

[57] J. Rodríguez-Laguna, L. Tarruell, M. Lewenstein, and A. Celi, Phys. Rev. A 95, 013627 (2017).

[58] A. Kosior, M. Lewenstein, and A. Celi, SciPost Phys. 5, 061 (2018).

[59] J. Louko, Classical Quantum Gravity 35, 205006 (2018).

[60] R.-Q. Yang, H. Liu, S. Zhu, L. Luo, and R.-G. Cai, Phys. Rev. Research 2, 023107 (2020).

[61] M. Dalmonte, B. Vermersch, and P. Zoller, Nat. Phys. 14, 827 (2018).

[62] G. Giudici, T. Mendes-Santos, P. Calabrese, and M. Dalmonte, Phys. Rev. B 98, 134403 (2018).

[63] V. Eisler and I. Peschel, J. Stat. Mech. (2018) 104001.

[64] F. Parisen Toldin and F. F. Assaad, Phys. Rev. Lett. 121, 200602 (2018).

[65] W. Zhu, Z. Huang, and Y.-C. He, Phys. Rev. B 99, 235109 (2019).

[66] X. Turkeshi, T. Mendes-Santos, G. Giudici, and M. Dalmonte, Phys. Rev. Lett. 122, 150606 (2019).

[67] T. Mendes-Santos, G. Giudici, M. Dalmonte, and M. A. Rajabpour, Phys. Rev. B 100, 155122 (2019).

[68] T. Mendes-Santos, G. Giudici, R. Fazio, and M. Dalmonte, New J. Phys. 22, 013044 (2020).

[69] J. Zhang, P. Calabrese, M. Dalmonte, and M. A. Rajabpour, SciPost Phys. Core 2, 007 (2020).

[70] I. Peschel, J. Phys. A: Math. Gen. 36, L205 (2003).

[71] I. Peschel and V. Eisler, J. Phys. A: Math. Theor. 42, 504003 (2009). 